\title{
Linde, Frank: Ist Schwarzkopieren eine Altersfrage? Eine Un- tersuchung zum Beschaffungsverhalten von digitalen Gütern in Abhängigkeit vom Alter. Köln: Fachhochschule Köln, Fakultät für Informations- und Kommunikationswissenschaften, Institut für Informationswissenschaft, 2014 (Kölner Arbeitspapiere zur Bibli- otheks- und Informationswissenschaft; 74) [Online unter: https:// www.fbi.fh-koeln.de/institut/papers/kabi/volltexte/band074.pdf]
}

„Mit zunehmendem Alter wird weniger schwarzkopiert“ (Linde, S. 7). Dieser (General-)Hypothese geht Frank Linde, Dozent am Institut für Informationswissenschaft an der Fachhochschule Köln, nach und versucht sich ihr einerseits über bereits vorhandenes Quellenmaterial, andererseits über eine Erhebung mittels Fragebogen zu nähern. Wer sich nun fragt, woher der eher untypische Begriff Schwarzkopieren stammt, wird durch eine Definition aufgeklärt: Der Terminus dient als Ersatz für die weit verbreitete Raubkopie, welche begrifflich gesehen eine Wegnahme und nicht die illegale Duplizierung bedeutet.

Im ersten Teil des Texts werden Kernaussagen der Sekundärliteratur herausgefiltert und die Auswertung durch eine Visualisierung der Objekte dargestellt. Diese Visualisierung dient als Basis für die Eingrenzung der Faktoren, welche mit den Stichworten Alter und Schwarzkopieren in Verbindung stehen, und damit der weiteren Hypothesenbildung. Im zweiten Block des Textes wird ausgehend vom theoretischen Unterbau eine empirische Studie anhand eines Fragebogens durchgeführt und analysiert.

30 Einflussfaktoren auf das Schwarzkopieren wurden von Linde gesammelt. Acht davon, welche mit dem Thema Alter in Verbindung stehen, wurden vom Autor aufgegriffen und für seine eigene Studie genutzt. Es wurden ausgehend von der Generalannahme, dass Alter des Individuums und Häufigkeit des Schwarzkopierens in Verbindung stehen, weitere sechs Hypothesen gebildet:

- Technische Fähigkeiten: Je höher die Kenntnisse und Fähigkeiten im dafür notwendigen Bereich sind, desto mehr wird schwarzkopiert.

- Berufliche Position: Je höher die berufliche Position, desto weniger wird schwarzkopiert.

- Zeitfaktor: Individuen kopieren weniger schwarz, je mehr Zeit sie für den Beruf aufbringen. 
- Kenntnisse der Rechtsverfolgung: Je höher das Grundverständnis der rechtlichen Lage, desto weniger wird schwarzkopiert.

- Moral \& Ethik: Es wird weniger schwarzkopiert, wenn die ethischmoralische Vorstellung höher eingestuft wird.

- Einkommen: Je höher das Einkommen, desto geringer ist das Schwarzkopierverhalten ausgeprägt.

Anhand der durchgeführten Befragungen bekräftigt Linde die Aussage, dass mit zunehmendem Alter das Schwarzkopierverhalten nachlässt. Weitere drei der sechs Zusatzhypothesen werden bestätigt. Problematisch ist dabei, dass die Argumentation nicht immer nachvollziehbar ist und teilweise tendenziös anmutet. So wird die Hypothese vom grundlegenden Zusammenhang von beruflicher Position und Schwarzkopieren widerlegt, bei der konkreten Abfrage des Verhaltens der letzten vier Monate aber bestätigt. Im Fazit wird diese Diskrepanz negiert und nur noch folgende Pauschalaussage getätigt: „Je höher die berufliche Position, desto weniger wird schwarzkopiert" (ebd., S. 34). Auch die Fragestellungen in der durchgeführten Untersuchung sind teilweise willkürlich. So werden unter der Bezeichnung „Berufstätigkeit“ einerseits Vollzeitanstellung, andererseits Führungsposition angeführt - zwei Variablen, die so nicht verglichen werden können! Der Einflussfaktor „Moral und Ethik“ wird mit der Frage nach Schuldgefühlen abgeprüft - einer subjektiven Einschätzung, die wohl kaum Vergleiche zulässt.

Auf das im ersten Teil ausgewertete Quellenmaterial wird im weiteren Verlauf kaum noch Bezug genommen. Das würde sich aber vor allem bei gegensätzlichen Ergebnissen anbieten: so zum Beispiel Aupperle 2010: „Mit höherem Alter [wird] mehr schwarzkopiert" (ebd., S. 12) vs. Linde 2014 „Mit zunehmendem Alter wird weniger schwarzkopiert" (ebd., S. 34).

Anhand der Quellen unterteilt der Autor die Informationsgüter in „Software“, „Musik“, „Filme“, „eBooks“, „Spiele“ und „Übergreifend“. Leider mangelt es hier an Klarheit in der Definition. An keiner Stelle im Text wird ausgeführt, dass es sich hierbei ausschließlich um digitale Informationsgüter handelt und physische Schwarzkopien ausgenommen sind. Die einzige Erwähnung hierzu findet sich im Titel. Überhaupt sind alle Definitionen im Text schwammig formuliert. Beim zentralen Thema, dem Schwarzkopieren, ist lapidar vom "geltenden Gesetz" die Rede. Auf welche Rechtsgrundlage hier Bezug genommen wird, wird nicht weiter angegeben. Wird auf Seite 2 noch ausgeführt, warum der Begriff „Schwarzkopieren“ verwendet werden soll, werden im weiteren Verlauf des Texts inkonsequenterweise Synonyme bzw. Umschreibungen wie ,illegale Downloads" verwendet. 
Der für einzelne Hypothesen herangezogene geringe Quellenpool und die sehr homogene Altersstreuung der Probanden des Fragebogens - der überwiegende Teil ist zwischen 18 und 35 Jahre alt - kann trotz dieser Ausarbeitung eine generelle Belegung der Generalhypothese, dass sich das Schwarzkopierverhalten indirekt proportional zum Alter verhält, in den Augen der Rezensentlnnen nicht zweifelsfrei bestätigen. Zu bemängeln ist weiters der im Unterpunkt 3.3 „Zielgruppen“ anklingende Umstand, dass für die Auswertung neben SchülerInnen hauptsächlich Personen aus dem universitätsnahen Umfeld herangezogen wurden - auf den Ausbildungsgrad der Probanden wird im Text jedoch nicht weiter eingegangen, obwohl im Fragebogen erwähnt. Gut aufbereitet ist hingegen die Faktorensammlung - welche Faktoren mit dem Thema Schwarzkopieren in Verbindung stehen -, besonders die Visualisierung dieser Sammlung ist positiv hervorzuheben.

Die Untersuchung „Ist Schwarzkopieren eine Altersfrage?" stellt eine Momentaufnahme dar. Diese soll zeigen, auf welche Art und Weise ein dynamischer Wert wie das Alter in Zusammenhang mit Einflussfaktoren wie Beruf, Freizeit oder Wissensstand, sich auf das Schwarzkopier-Verhalten auswirken kann. Problematisch an dieser Momentaufnahme ist der fehlende Blick in die Zukunft. Wie sehen die Umfrageergebnisse aus, wenn die heute jungen Schwarzkopierenden älter werden? Wird es ein allgemeines gesellschaftliches Umdenken geben? Nur in dieser Art Kontext ist auch die vorliegende Veröffentlichung zu sehen: Sie richtet sich an ein Fachpublikum, das aufbauend auf diese Studie weitere Untersuchungen vornehmen kann. Die Untersuchung zum Beschaffungsverhalten von digitalen Gütern in Abhängigkeit vom Alter ist ein weiterer kleiner Baustein im Forschungsgebiet der Informationswissenschaft - mehr auch nicht.

Magdalena Andrae, Salzburg \& Magdalena Zelger, Wien

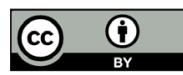

Dieses Werk ist lizenziert unter einer Creative-Commons-Lizenz Namensnennung 4.0 International 